\title{
铁酸钾 $\left(\mathrm{KFeO}_{2}\right)$ 热稳定性的穆斯堡尔研究
}

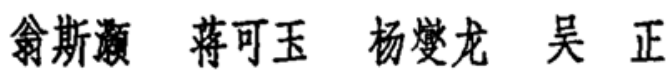 \\ (华东师范大学物理系,上海 200062)
}

\section{关词 $\mathrm{KFeO}_{2}$ 、热分解、穆斯堡尔效应}

固体化合物的化学反应或含有固体的多相催化反应通常是一个复杂的过程. 要了解其转 化特性和确定其中间产物往往是相当困难的. X衍射分析技术是一种常用的有效方法, 但如 果被测样品(产物)是非晶或微晶颗粒, 谱图上会出现很宽的谱线, 甚至导致特征谱线的消失. 穆斯堡尔谱学方法能在原子水平上提供许多有价值的信息, 并可在反应条件下进行实地观察, $\mathrm{KFeO}_{2}$ 热分解产物的分析就是这样一个例子. 由于 $\mathrm{KFeO}_{2}$ 在一些催化反应中起着重要的作 用, 因而研究反应条件下 $\mathrm{KFeO}_{2}$ 的存在形态及其与催化活性的关系等是人们颇为关心的重要 课题. 本文用穆斯堡尔谱学方法对 $\mathrm{KFeO}_{2}$ 在制备过程中的热稳定性进行考察, 发现了一些有 意义的结果,为解决上述问题提供了有用的信息.

\section{一、实 检}

$\mathrm{KFeO}_{2}$ 用 $\alpha-\mathrm{FeOOH}$ 和 $\mathrm{K}_{2} \mathrm{CO}_{3}$ 粉末, 经研磨、压片, 在 600-700年焙烧 $10 \mathrm{~h}$ 烧结而成. 边焙烧边测量用的穆斯堡尔高温炉用石英玻璃制成，窗口厚度小于 $100 \mu \mathrm{m}$, 对, $5 \mathrm{Fe} 14.4 \mathrm{keV} \gamma$ 射线的透过无明显影响. 温度控制采用 CW-701 控温仪. 纵向磁场用永久磁铁获得. 放射 源 ${ }^{7} \mathrm{Co}(\mathrm{Rh})$ 约 $40 \mathrm{mCi}$. 用 $25 \mu \mathrm{m}$ 厚的 $\alpha-\mathrm{Fe}$ 簿进行速度定标. 所有穆斯堡尔谱参数均经计 算机拟合. 文中化学移位指相对于 $\alpha-\mathrm{Fe}$ 谱中心而言.

\section{二、结果与讨论}

图 1(a) 和 (b) 分别是 $\mathrm{KFeO}_{2}$ 在室温和高温 $700^{\circ} \mathrm{C}$ 时的穆斯堡尔谱. 室温谱旺磁分裂, 其超精细场 $H=500 \mathrm{k}(1000 / 4 \pi) \mathrm{A} / \mathrm{m}$, 化学移位 $\delta-0.21 \mathrm{~mm} / \mathrm{s}$, 与文献[1]报道的相近. 高 温 $\left(700^{\circ} \mathrm{C}\right)$ 谱仍保留部分磁分裂, 说明 $\mathrm{KFeO}_{2}$ 是具有很高 $\mathrm{Neel}$ 温度的反铁磁性化合物, 其 $T_{N}=983 \mathrm{~K}^{[2]}$. 利用 $\mathrm{KFeO}_{2}$ 具有比其它一般铁氧化物更高的磁相变温度, 就可以鉴别它的生 成和存在.

图 2(a)-(d) 是 $\mathrm{KFeO}_{2}$ 在室温下, 暴露于空气中经过不同时间间隔所获得的穆斯堡尔 谱.图 2(a) 为 $12 \mathrm{~h}$ 以后测得的谱, 6 线中间出现一组四极分裂双峰, 化学移位 $\delta-0.19 \mathrm{~mm} / \mathrm{s}$, 四极分裂 $\Delta=0.89 \mathrm{~mm} / \mathrm{s}$. 随着时间的推移, 其顺磁部分增大 (图 2(b)), 直至以后 6 线全部 消失 (图 2(c)). 这说明 $\mathrm{KFeO}_{2}$ 在室温下极易分解, 成为非晶态小颗粒而呈现超顼磁性. 正 因为这个原因, 致使以往 $\mathrm{X}$ 行射分析未能观察到它的存在 ${ }^{\text {. }}$. 这也许可以解释一些作者关于 标准的 $\mathrm{KFeO}_{2}$ 谱线是双线还是 6 线不同观点的讨论 ${ }^{(3)}$. 使人惊奇的是 $\mathrm{KFeO}_{2}$ 在空气中放置

本文 1989 年 6 月28日收到. 1989 年 9 月 1 日收到密改稍. 


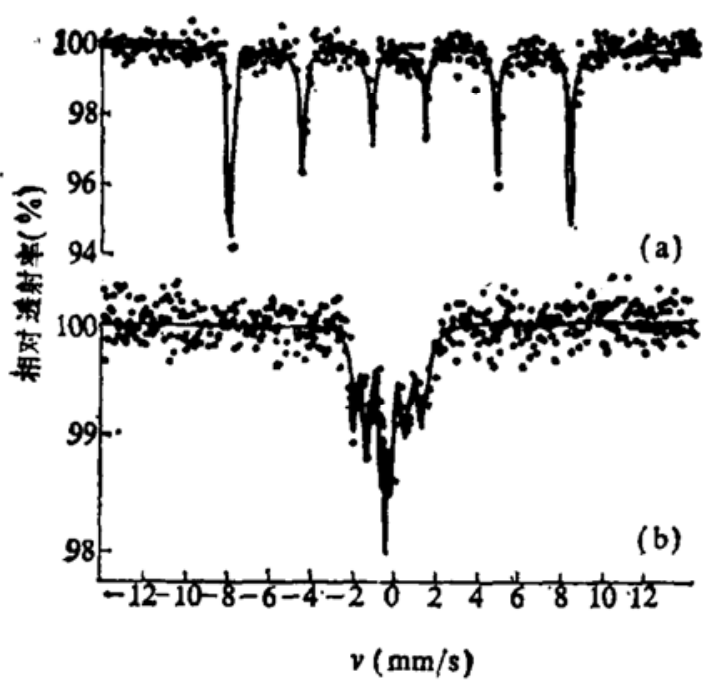

图 $1 \mathrm{KFeO}_{2}$ 的穆斯堡尔谱

(a) 室温； (b) $700^{\circ} \mathrm{C}$

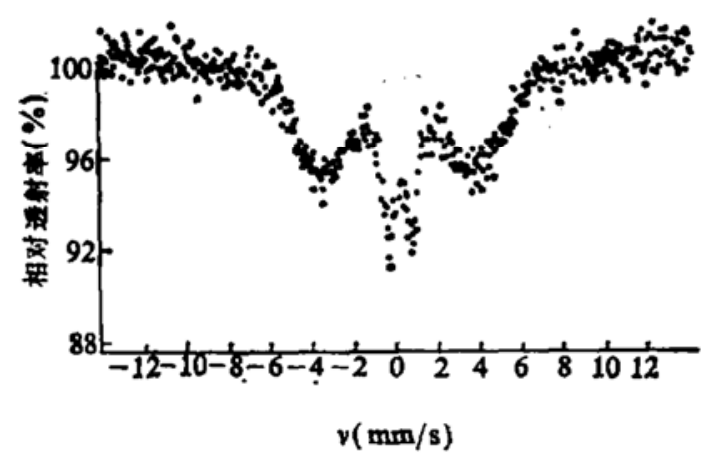

图 $3 \mathrm{KFeO}$ ，分解的最后产物加 $10 \mathrm{k}(1000 / 4 \pi) \mathrm{A} / \mathrm{m}$ 纵 向磁场后的穆斯堡尔谱

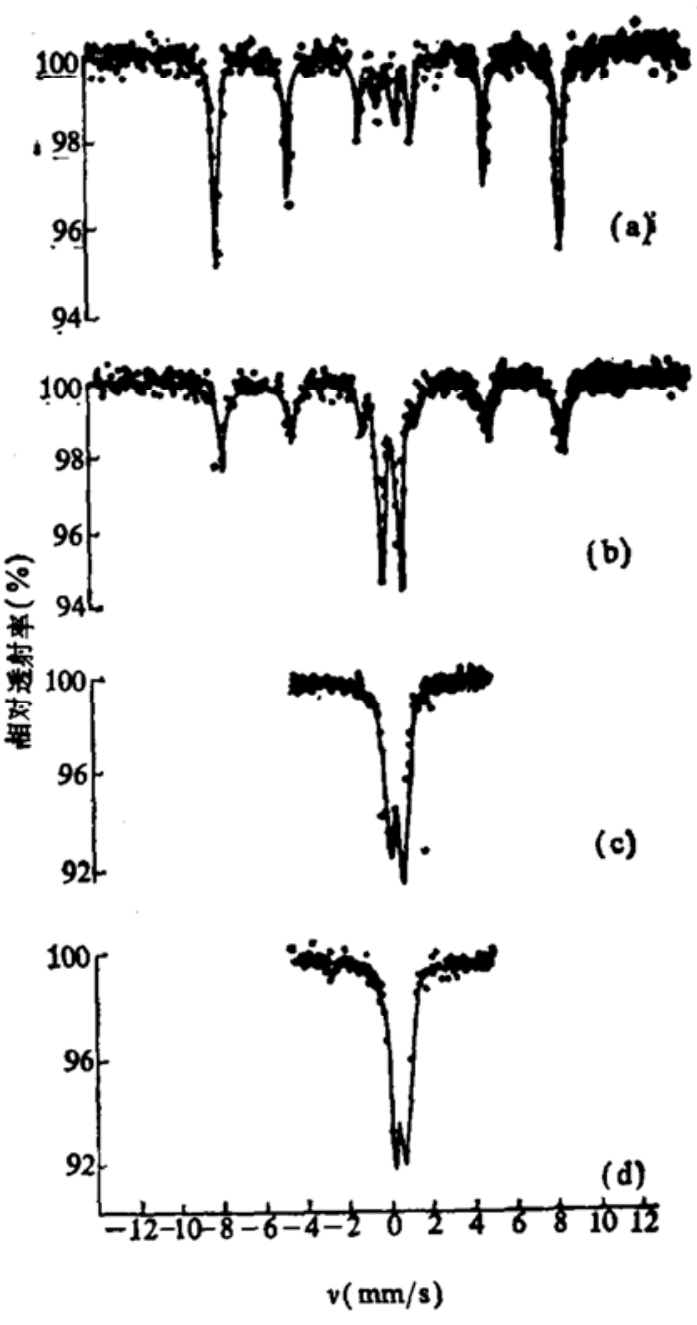

图 $2 \mathrm{KFeO}_{2}$ 在室温下暴露于空气中经过 不同 时间后获得的穆斯堡尔谱
(a) $12 \mathrm{~h}$;
(b) $31 \mathrm{~h}$;
(c) 16 天;
(d) 36 天

16 天以后, 原来的顺磁峰继续发生变化（图 2(c)、(d)), 出现一组四极分裂更小的双线并逐 渐取代原有的谱线, 其 $\Delta-0.55 \mathrm{~mm} / \mathrm{s}, \delta-0.31 \mathrm{~mm} / \mathrm{s}$. 为了进一步鉴别该产物的特性, 对 图 2(d) 谱所示样品加 $10 \mathrm{k}(1000 / 4 \pi) \mathrm{A} / \mathrm{m}$ 纵向磁场, 发现谱线出现磁分裂, 其 $2 、 5$ 线消失, $1 、 6$ 线很宽，呈现典型的非晶颗粒谱线的特征 (图 3). 此时样品的颜色由 $\mathrm{KFeO}_{2}$ 所具有的 青绿色转为橙灰色, 并有弱磁性. 参考文献 [4] 的数据, 我们推测该小颗粒为 $\gamma-\mathrm{Fe}_{2} \mathrm{O}_{3}$, 样 品表面及样品架上出现的白色粉末是析出的 $\mathrm{K}_{2} \mathrm{O}$.

综上所述, $\mathrm{KFeO}_{2}$ 在空气中是不稳定的化合物, 它极易吸湿并很快转变为 $\boldsymbol{r}-\mathrm{Fe}_{2} \mathrm{O}_{3}$ 的小 颗粒和 $\mathrm{K}_{2} \mathrm{O}$. 由于它的高 Néel 温度以及特定的分解过程和产物, 使得在研究 $\mathrm{KFeO}_{2}$ 对某 些催化反应的作用时,穆斯堡尔方法可以作为一种有效的分析手段。

\section{考文嗝}

[1] Toshio Ichida, Bull. Chem. Soc. Japan, 46(1973),79.

[2] Toshio Ichida et al., J. Phys. Soc. Japan, 29(1970), 1109.

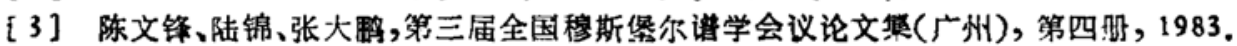

(4) Krupyankii, Y.F. and Suzdalev, I.P., Sov. Phys. JETP, 38 (1974), 859. 